\title{
Growth characteristics of different heart cells on novel nanopatch substrate during electrical stimulation
}

\author{
David A. Stout ${ }^{\mathrm{a}, \mathrm{b}, *}$, Emilia Raimondo ${ }^{\mathrm{c}}$, Giuliano Marostica ${ }^{\mathrm{a}}$ and Thomas J. Webster ${ }^{\mathrm{d}}$ \\ ${ }^{\text {a } C e n t e r ~ f o r ~ B i o m e d i c a l ~ E n g i n e e r i n g, ~ S c h o o l ~ o f ~ E n g i n e e r i n g, ~ B r o w n ~ U n i v e r s i t y, ~} 184$ Hope St., Box D, \\ Providence RI 02906, USA \\ ${ }^{\mathrm{b}}$ Department of Mechanical and Aerospace Engineering, California State Univeristy, Long Beach, \\ 1250 Bellflower Blvd., Long Beach CA 90840, USA \\ ${ }^{\mathrm{c}}$ Division of Biology and Medicine, Brown University, 91 Waterman Street Providence RI 02912, USA \\ ${ }^{\mathrm{d}}$ Department of Chemical Engineering, Northeastern University, 360 Huntington Ave., Boston MA \\ 02115, USA
}

\begin{abstract}
During a heart attack, the heart's oxygen supply is cut off, and cardiomyocytes perish. Unfortunately, once these tissues are lost, they cannot be replaced and results in cardiovascular disease-the leading cause of deaths worldwide. Advancements in medical research have been targeted to understand and combat the death of these cardiomyocytes. For example, new research (in vitro) has demonstrated that one can expand cardiomyocyte adhesion and proliferation using poly lactic-co-glycolic acid (PLGA) (50:50 (weight percent)) supplemented with carbon nanofibers (CNFs) to create a cardiovascular patch. However, the examination of other cardiovascular cell types has not been investigated. Therefore, the purpose of this present in vitro study was to determine cell growth characteristics of three different important cardiovascular cell types (aortic endothelial, fibroblast and cardiomyocyte) onto the substrate. Cells were seeded onto different PLGA:CNF ratio composites to determine if CNF density has an effect on cell growth, both in static and electrically stimulated environments. During continuous electrical stimulation (rectangle, $2 \mathrm{~nm}, 5 \mathrm{~V} / \mathrm{cm}, 1 \mathrm{~Hz}$ ), cardiomyocyte cell density increased in comparison to its static counterparts after 24, 72 and 120 hours. A minor rise in Troponin I excretion in electrical stimulation compared to static conditions indicated nominal cardiomyocyte cell function during cell experiments. Endothelial and fibroblast cell growth experiments indicated the material hindered or stalled proliferation during both static and electrical stimulation experiments, thus supporting the growth of cardiomyocytes onto the dead tissue zone. Furthermore, the results specified that CNF density did have an effect on PLGA:CNF composite cytocompatibility properties with the best results coming from the 50:50 [PLGA:CNF (weight percent:weight percent)] composite. Therefore, this study provides further evidence that a conductive scaffold using nanotechnology should be further research for various cardiovascular applications.
\end{abstract}

Keywords: Nanomaterials, cardiomyocyte, fibroblast, cardiovascular patch, carbon nanofiber

\footnotetext{
${ }^{*}$ Corresponding author: David A. Stout, Department of Mechanical and Aerospace Engineering, California State University, Long Beach, 1250 Bellflower Blvd., Long Beach CA 90840, USA. Tel.: +1 562985 1502; Fax: +1 562985 4408; E-mail: david_stout@brown.edu/david.stout@csulb.edu.
}

0959-2989/14/\$27.50 @ 2014 - IOS Press and the authors. 


\section{Introduction}

A myocardial infarction, commonly referred as a heart attack, takes place when the heart's supplementary blood vessels are obstructed by an unstable buildup within the vessels. Without blood flow and oxygen, special heart cells (cardiomyocytes) perish. This causes whole portions of cardiac tissue to die. Unfortunately, once these tissues are lost, they cannot be replaced. Additionally, fibrosis or cells which do not contract may take place within the void and causing permanent damage-the main cause of cardiovascular disease (CVD) [1].

Recent understanding to what causes a myocardial infarction has lead to improved ways to combat such a deadly disease and improved health of patients with CVD. Unfortunately, the progression of CVD in the world is still growing [2]. For this reason, new methods to recognize and alleviate CVD in asymptomatic patients are necessary to inhibit the first symptoms of CVD from being the last.

Regrettably, no single scientific field presents the perfect answer in fighting the advancement of CVD. Fortunately, the combination of multiple biological fields-cell biology, physiology and histology-joined with new advancements in material sciences and engineering at the nanoscale level (one physical dimension between 1-100 nm) have spawned a new field of medicine, known as nanomedicine [3].

The introduction of these "nanomaterials," from the advent of nanomedicine, has revolutionized the field of biomedical research. Due to their range of different medical applications, ranging from batteries and electronics to medicine [4,5], nanomaterials are among the most comprehensively examined materials to date. Furthermore, the change in the material's property at the nano level-improved mechanical, physical and chemical properties-gives nanomaterials the ability to be adaptable for a wide range of applications.

One recent advancement of nanomedicine is the combination of poly lactic-co-glycolic acid (PLGA) with embedded carbon nanofibers (CNF) to construct a conductive composite "patch" for the dead tissue zone (infarct area) of the heart. Studies have shown that when one adds CNFs to PLGA, cardiomyocytes (specific heart tissue cells responsible for contraction) will attach and propagate greater than on their non-nanoreinforced non-conductive counterparts [5]. Unfortunately, the mechanism and other heart cell interactions on the substrate is unknown.

To understand cell function on a novel nanomaterial, the purpose of this present in vitro study was to determine cell growth characteristics of three different cell types; aortic endothelial, fibroblast and cardiomyocyte. Cells were seeded onto different PLGA:CNF ratio composites to determine if CNF density has an effect on a nano-inspired cardiovascular patch, both in static and electrically stimulated environments.

\section{Materials and methods}

\subsection{Cardiovascular patch construction}

Fabrication of the nanomaterial cardiovascular patch started by measuring $500 \mathrm{mg}$ of PLGA (50:50 percent weight polylactic acid: polyglycolic acid) which was diluted in a $20 \mathrm{~mL}$ disposable scintillation vial with $20 \mathrm{~mL}$ of chloroform (Fisher Science) and then water bath sonicated below $30^{\circ} \mathrm{C}$ for $45 \mathrm{~min}$. Next, $500 \mathrm{mg}$ of CNFs (99.9\% pure by percent weight, Catalytic) with a diameter of $100 \mathrm{~nm}$ and lengths of 100-200 $\mu \mathrm{m}$ were diluted in a $50 \mathrm{~mL}$ beaker with $30 \mathrm{~mL}$ of chloroform (Fisher Science) and ultra sonicated (Misonix Sonicator 3000) at 20W for $30 \mathrm{~min}$. 


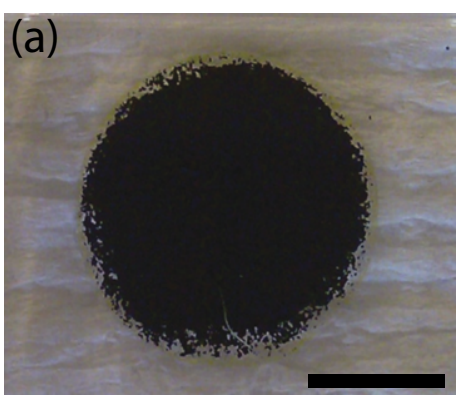

(c)

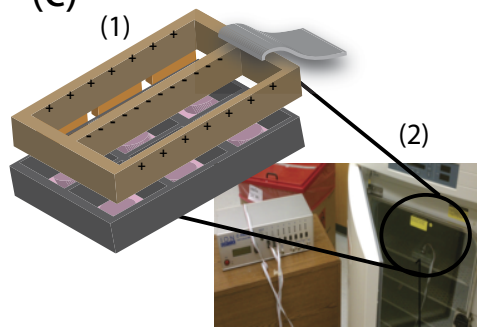



(d)

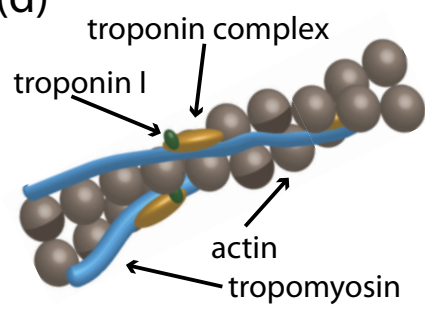

Fig. 1. Material analysis of a 50:50 [PLGA:CNF (weight:weight)] composite. a) Image of nanopatch composite after all synthesis steps were completed. Scale bar $=10 \mathrm{~mm}$. b) Scanning electron micrograph at $1 \mathrm{~K}$ magnification showing the distribution of CNFs in a PLGA matrix. Scale bar $=20 \mu \mathrm{m}$. (c) A (1) schematic of the C-Pace EP Culture Pacer where samples with cell media (pink) are added to the bottom 6-well holder (grey). Next, the C-Pace EP Culture Pacer top (gold) is placed on top of bottom well and connected to an electrical system. (c)(2) System is placed into incubator where the experiment starts. (d) A schematic of the Troponin complex which consists of three subunits: Troponin C (TnC), Troponin I (TnI) (shown), and Troponin $\mathrm{T}$ (TnT). As calcium binds to Troponin $\mathrm{C}$, it causes conformational changes that lead to disconnection of Troponin I. This allows tropomyosin to leave the binding site for myosin on actin leading to the contraction of the muscle.

After separate PLGA and CNF solutions were obtained, they were combined in $20 \mathrm{~mL}$ disposable scintillation vials at specific weight ratios ([PLGA:CNF] 100:0, 75:25, 50:50, 25:75, 0:100). Next, each composite solution was again water bath sonicated for $30 \mathrm{~min}$ to generate a homogeneous non-clumpy solution.

For experimental ease, $22 \mathrm{~mm}$ diameter microscope coverslips (Fisher Science) were coated with the composite solutions. Prior to coating, the glass substrates were disinfected by submerging in 70:30 (volume percent) ethanol-deionized water while shaking (VWR) for $10 \mathrm{~min}$. Next, the coverslips were autoclaved for $1 \mathrm{hr}$ on dry-cycle. Disposable pipettes (Fisher Science) were used to coat each glass substrate with the appropriate composite solution. The coated substrates were then vacuum dried (Shel Lab) at $205 \mathrm{kPa}$ vacuum pressure for $48 \mathrm{hr}$ for chloroform evaporation. Prior to cell experiments, samples were cleaned via a 10 min ethanol-deionized water (70:30 (volume percent)) soaking and then an overnight ultraviolet light sterilization.

\subsection{Evaluation of cell type proliferation under static and electrical stimulation conditions}

Three types of cells were used in this study; rat aortic endothelial cells (VEC Technologies), rat fibroblasts (ATCC: NIH/3T3 cells) and cardiomyocytes (Celprogen). Rat fibroblast and aortic endothelial cells were cultured in 6-well cell culture plates (Corning) and maintained with Dulbeccos's modified Eagle Medium (DMEM-High Glucose) supplemented with 10\% fetal bovine serum (FBS) and 1\% penicillinstreptomycin, at standard incubation conditions $\left(5 \% \mathrm{CO}_{2}, 95 \%\right.$ humidified air at $\left.37^{\circ} \mathrm{C}\right)$. Cardiomyocytes were cultured onto a 6-well cardiomyocyte cell culture extracellular matrix dish (Celprogen) and main- 

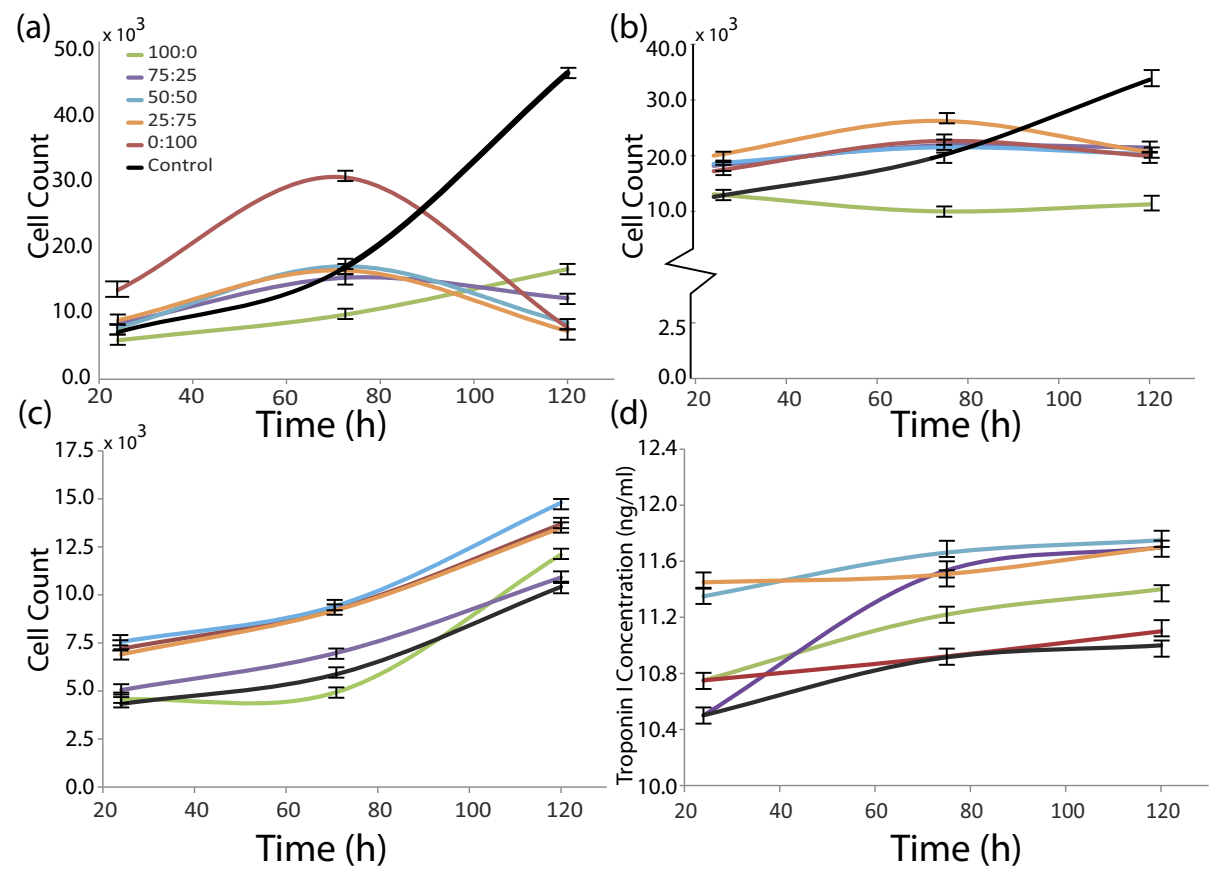

Fig. 2. Cell proliferation patterns under static conditions. Results of (a) rat aortic endothelial cells, (b) rat fibroblast cells, (c) cardiomyocytes and (d) Troponin I concentration in cardiomyocyte media was completed at 24, 72 and 120 hr under standard incubation conditions. Data are mean counts \pm s.d. to $\mathrm{n}=3$. Control is an etched glass coverslip.

tained with complete growth media cardiomyocyte media (Celprogen) enriched with 10\% FBS and 1\% penicillin-streptomycin at standard incubation conditions.

Single-cell type proliferation assays were performed in two different conditions: static and electrical stimulation conditions. For static conditions, each cell type was seeded at a density of $10^{4}$ cells $/ \mathrm{mL}$, on the various aforementioned PLGA:CNF density samples (with a glass etched coverslip acting as a control) in a 6-well rectangular cell culture dish (Corning). In each case, the samples and their appropriate media were incubated for 24,72 and $120 \mathrm{hr}$ under standard incubation conditions, changing the media every other day.

For single-cell type proliferation assay under electrical stimulation conditions, each cell type was seeded at a density of $10^{4}$ cells $/ \mathrm{mL}$, on top of the various PLGA:CNF density samples (with a glass etched coverslip acting as a control) in a 6-well rectangular cell culture dish (Corning). Again, the samples and appropriate media were continuously stimulated (rectangle signal, $2 \mathrm{~nm}, 5 \mathrm{~V} / \mathrm{cm}, 1 \mathrm{~Hz}$ ) with a C-Pace EP Culture Pacer (IonOptix) for 24, 72 and $120 \mathrm{hr}$ to mimic normal heart performance [7] (Figure 1c).

At the end of each time interval, samples were transferred to new 6-well tissue culture plates (Corning). $1 \mathrm{~mL}$ of the appropriate media for each cell type was added and $200 \mu \mathrm{L}$ of MTS dye solution (Promega CellTiter 96 AQueous Assay) was added to each well and incubated for $4 \mathrm{hr}$ under standard incubator conditions. Each solution was then transferred to 96-well plates (BD Falcon) and optical density values were measured using a spectrophotometer (Molecular Devices) to measure and analyze cytocompatibility and cell viability on the composite.

To evaluate cardiomyocyte contract ability, Troponin I enzyme-linked immunosorbent assay (ELIZA) (Raybiotech) were also performed on cardiomyocyte media (Figure 1d). 

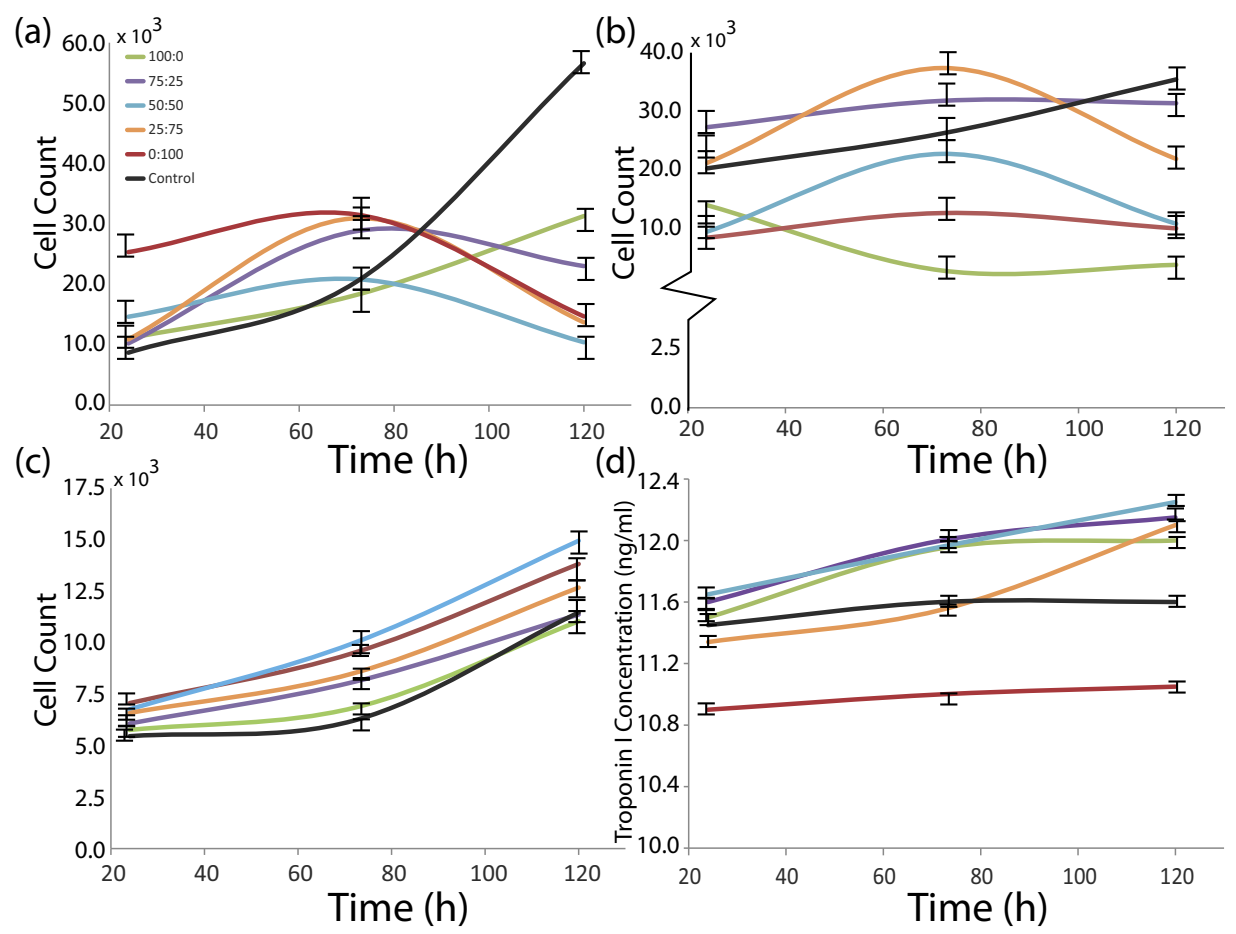

Fig. 3. Cell proliferation patterns under continuous stimulation (rectangle, $2 \mathrm{~nm}, 5 \mathrm{~V} / \mathrm{cm}, 1 \mathrm{~Hz}$ ). Results of (a) rat aortic endothelial cells, (b) rat fibroblast cells, (c) cardiomyocytes and (d) Troponin I concentration in cardiomyocyte media was completed at 24, 72 and $120 \mathrm{hr}$ under standard incubation conditions. Data are mean counts \pm s.d. to $\mathrm{n}=3$. Control is an etched glass substrate.

\subsection{Statistical analysis}

All proliferation experiments were performed in triplicate with three repeats each with an etched glass coverslip acting as a control. Data was subjected to the chi-square goodness-of-fit test to determine if the data sample comes from a normal distribution. The optical density (OD) data were plotted as the mean \pm standard error of the mean while OD to cell count conversions was conducted by using the four parameter logistic (4PL) equation between OD and cell numbers. When data were compared, Wilcoxon rank-sum, one-way ANOVA and a student T-test were used for comparison of the means. P-value of $<$ 0.05 was considered to be significant.

\section{Results \& discussion}

\subsection{Material fabrication}

A SEM micrograph of a PLGA:CNF composite surface shown in Figure 1b. As seen, CNFs were spread throughout the PLGA matrix, implying that the composites were accurately produced (Figure 1a).

\subsection{PLGA:CNF cytocompatibility and cell growth}

For cardiomyocyte growth experiments, results inferred that a PLGA:CNF composite can promote cardiomyocyte proliferation and growth superior when electrically stimulated compared to a PLGA ma- 
Table 1

Summary of change in cell growth

\begin{tabular}{|c|c|c|c|c|c|c|c|}
\hline \multirow[t]{3}{*}{ Cell Type } & \multirow{2}{*}{\multicolumn{4}{|c|}{$\begin{array}{l}\text { [PLGA:CNF] } \\
\text { sample }\end{array}$}} & owth $(\% \pm$ s.d & & \\
\hline & & & & & \multicolumn{3}{|c|}{ Electrical stimulation } \\
\hline & & $24-72 \mathrm{hr}$ & $72-120 \mathrm{hr}$ & $24-120 \mathrm{hr}$ & $24-72 \mathrm{hr}$ & $72-120 \mathrm{hr}$ & $24-120 \mathrm{hr}$ \\
\hline \multirow[t]{6}{*}{ Endothelial } & $100: 0$ & $64.9 \pm 5.2$ & $71.6 \pm 5.1$ & $182.9 \pm 9.1$ & $68.8 \pm 5.5$ & $72.1 \pm 2.5$ & $187.2 \pm 3.2$ \\
\hline & $75: 25$ & $83.1 \pm 4.2$ & $-20.0 \pm 5.1$ & $40.0 \pm 5.7$ & $181.7 \pm 10.2$ & $-20.0 \pm 5.6$ & $125.2 \pm 9.5$ \\
\hline & $50: 50$ & $119.2 \pm 8.5$ & $-50.1 \pm 4.5$ & $9.3 \pm 4.2$ & $42.5 \pm 3.2$ & $-50.1 \pm 4.7$ & $-28.8 \pm 4.1$ \\
\hline & $25: 75$ & $84.7 \pm 5.7$ & $-55.7 \pm 4.6$ & $-18.1 \pm 1.4$ & $84.2 \pm 4.2$ & $-59.5 \pm 4.8$ & $-10.8 \pm 3.1$ \\
\hline & $0: 100$ & $126.5 \pm 8.4$ & $-74.4 \pm 4.5$ & $-42.0 \pm 4.5$ & $24.6 \pm 2.2$ & $-53.4 \pm 3.8$ & $-42.0 \pm 5.1$ \\
\hline & Control & $134.9 \pm 9.5$ & $150.5 \pm 8.7$ & $301.1 \pm 11.1$ & $134.9 \pm 10.0$ & $141.0 \pm 5.5$ & $350.0 \pm 12.1$ \\
\hline \multirow[t]{6}{*}{ Fibroblast } & 100:0 & $-9.2 \pm 2.5$ & $4.1 \pm 2.1$ & $-5.0 \pm 1.9$ & $-32.2 \pm 5.3$ & $4.1 \pm 2.1$ & $-29.4 \pm 4.1$ \\
\hline & $75: 25$ & $9.3 \pm 2.2$ & $-0.7 \pm 2.1$ & $8.5 \pm 1.1$ & $9.3 \pm 2.4$ & $-0.7 \pm 1.2$ & $8.5 \pm 0.8$ \\
\hline & $50: 50$ & $7.6 \pm 1.4$ & $-2.9 \pm 0.8$ & $4.4 \pm 1.4$ & $44.1 \pm 5.4$ & $-27.5 \pm 3.1$ & $4.4 \pm 1.1$ \\
\hline & $25: 75$ & $15.5 \pm 2.1$ & $-12.1 \pm 1.7$ & $1.5 \pm 0.7$ & $38.6 \pm 4.9$ & $-26.7 \pm 3.6$ & $1.5 \pm 0.5$ \\
\hline & $0: 100$ & $14.4 \pm 2.5$ & $-6.1 \pm 1.1$ & $7.3 \pm 2.4$ & $14.4 \pm 2.5$ & $-7.8 \pm 1.4$ & $5.4 \pm 2.1$ \\
\hline & Control & $21.2 \pm 2.4$ & $35.3 \pm 3.1$ & $64.5 \pm 4.1$ & $14.5 \pm 2.1$ & $19.7 \pm 3.6$ & $37.1 \pm 4.4$ \\
\hline \multirow[t]{6}{*}{ Cardiomyocyte } & 100:0 & $10.0 \pm 1.4$ & $60.0 \pm 4.4$ & $71.1 \pm 2.4$ & $18.6 \pm 3.5$ & $61.4 \pm 5.4$ & $91.6 \pm 6.5$ \\
\hline & $75: 25$ & $30.0 \pm 3.4$ & $46.2 \pm 3.4$ & $90.1 \pm 4.4$ & $34.6 \pm 2.9$ & $45.3 \pm 2.4$ & $95.6 \pm 2.4$ \\
\hline & $50: 50$ & $25.9 \pm 2.7$ & $55.4 \pm 3.4$ & $95.8 \pm 3.4$ & $47.7 \pm 2.3$ & $49.6 \pm 2.1$ & $121.5 \pm 3.5$ \\
\hline & $25: 75$ & $34.4 \pm 2.6$ & $45.3 \pm 2.9$ & $95.4 \pm 2.4$ & $29.3 \pm 3.3$ & $48.7 \pm 3.1$ & $92.4 \pm 2.2$ \\
\hline & $0: 100$ & $39.5 \pm 3.4$ & $54.9 \pm 3.3$ & $116.2 \pm 5.0$ & $33.3 \pm 1.4$ & $40.3 \pm 3.1$ & $89.1 \pm 2.1$ \\
\hline & Control & $37.3 \pm 3.1$ & $75.5 \pm 3.6$ & $140.9 \pm 11.0$ & $13.9 \pm 2.3$ & $84.3 \pm 2.8$ & $150.1 \pm 6.0$ \\
\hline
\end{tabular}

terial with no CNFs (Figure 2c, 3c). More importantly, an increase in cardiomyocyte growth between all time points were shown for both static and electrical stimulation (Table 1) experiments. Universal growth trends were detected between each PLGA:CNF ratio and support previously published results [8].

Looking at cardiomyocyte function, Troponin I assays determined that during cardiomyocyte cytocompatibility experiments, cardiomyocytes had a functional Troponin complex. Interestingly, it was shown that most of all CNF experiments for all time points exhibited a minor, but significant increase of Troponin I, demonstrating better productivity [9]. More importantly, the Troponin I assays detected similar trends shown in the both static and electrical stimulation cell growth cytocompatibility assays (Figure $2 d, 3 d)$.

Interestingly, results indicate endothelial and fibroblast cell growth were hindered or stalled during both static and electrical stimulation experiments (Table 1). When CNFs were added to the material, a general trend was observed for both cell types in both environments; an increase in cell density up to 72 $\mathrm{hr}$, then a decrease in cell growth for an overall small increase in cell growth (Figure 2, 3). These results may help in supporting the growth of cardiomyocytes onto the dead tissue zone and decrease fibrosis or non-contractile cell growth within the infarct area.

It is important to note other studies have shown by using nanotechnology [4] or stem cells [10] for cardiovascular applications can promote healthy tissue growth for an infarct myocardial area, but becomes very laborious, and growth of other cells are unknown. By creating a simple-solution system with the use of nanotechnology, we are able to overcome some of these hurdles and add to the knowledge, that nanotechnology may have the ability to hinder fibrosis or non-contractile cell growth.

Why a greater amount of cardiomyocyte attachment and growth were noticed on nano-composites with increasing CNF density in PLGA while endothelial and fibroblast cells did not is not known, but 
material research may assist in understanding its mechanism. Research in material science and biology have established that the adsorption and bioactivity of important cell binding proteins (like vitronectin and laminin) increase on nanophase ceramics and materials were due to roughness values closer to the nanometer roughness of natural tissue itself [11]. Also, materials can be too rough or toxic and can impede cellular movement and activity [12]-a mechanism that may be seen during endothelial and fibroblast experiments.

Even though the mechanism is not fully understood, it is shown here that CNF density does play a role in mediating cardiovascular cell type growth and functionality. This may be due to the creation of a material which resembles normal heart tissue material characteristics better (such as topography, conductivity, mechanical properties, etc.).

\section{Conclusion}

This work demonstrates that a PLGA:CNF composite, with the use of electrical stimulation to imitate a normal heart environment, can stimulate cardiomyocyte growth and activity while hindering fibroblast and endothelial cell growth. Using an electrical stimulation system, showed that an increase in cardiomyocyte density (growth) and Troponin I can be achieved on all PLGA:CNF composite ratios, when related to non-electrical stimulation data and control substrates. These results indicate that CNF density does have an effect on PLGA:CNF composite cytocompatibility. Also, it can be continually investigated for cardiovascular tissue engineering applications-where $100 \mathrm{~nm}$ CNF 50:50 PLGA:CNF (weight:weight) composites would be the leading ratio for future studies.

\section{References}

[1] R. Khan and R. Sheppard, Fibrosis in heart disease: understanding the role of transforming growth factor- $\beta 1$ in cardiomyopathy, valvular disease and arrhythmia, Immunology 118 (2002), 10-24.

[2] B.J. Gersh, K. Sliwa, B.M. Mayosi and S. Yusuf, The epidemic of cardiovascular disease in the developing world: global implications, European Heart Journal 31 (2010), 642-648.

[3] R. Valiev, Materials science: Nanomaterial advantage, Nature 419 (2002), 887-889.

[4] T. Dvir, B.P. Timko, M.D. Brigham, S.R. Naik and S.S. Karajanagi, Nanowired three-dimensional cardiac patches, Nature Nano 720 (2011), 720-725.

[5] D.A. Stout, B. Basu and T.J. Webster, Poly(lactic-co-glycolic acid): Carbon nanofiber composites for myocardial tissue engineering applications, Acta Biomaterialia 7 (2011), 3101-3112.

[6] A. Verma and F. Stellacci, Effect of Surface Properties on Nanoparticle-Cell Interactions, Small 6 (2010), 12-21.

[7] Y. Xia, L.M. Buja and J.B. McMillin, Change in Expression of Heart Carnitine Palmitoyltransferase I Isoforms with Electrical Stimulation of Cultured Rat Neonatal Cardiac Myocytes, Journal of Biological Chemistry 271 (1996), 12082-12087.

[8] D.A. Stout, J. Yoo, A.N. Santiago-Miranda and T.J. Webster, Mechanisms of greater cardiomyocyte functions on conductive nanoengineered composites for cardiovascular application, International Journal of Nanomedicine 7 (2012), 5653-5669.

[9] A.T. Soufan, G. van den Berg, J.M. Ruijter, P.A.J. de Boer and M. J. B. van den Hoff, Regionalized Sequence of Myocardial Cell Growth and Proliferation Characterizes Early Chamber Formation, Circulation Research 99 (2006), 545-552.

[10] P. Lee, M. Klos, C. Bollensdorff, L. Hou, P. Ewart, T.J. Kamp, J. Zhang, A. Bizy, G. Guerrero-Serna, P. Kohl, J. Jalife and T.J. Herron, Simultaneous Voltage and Calcium Mapping of Genetically Purified Human Induced Pluripotent Stem Cell-Derived Cardiac Myocyte Monolayers, Circulation Research 110 (2012), 1556-1563.

[11] T.J. Webster, C. Ergun, R.H. Doremus, R.W. Siegel and R. Bizios, Specific proteins mediate enhanced osteoblast adhesion on nanophase ceramics, Journal of Biomedical Materials Research 51 (2000), 475-479.

[12] L. Yang, B.W. Sheldon and T.J. Webster, The impact of diamond nanocrystallinity on osteoblast functions, Biomaterials 30 (2009), 3458-3462. 